\title{
Coagulation factor III (tissue factor) is required for vascularization in zebrafish embryos
}

\author{
R.F. Zhou, Y. Liu, Y.X. Wang, W. Mo and M. Yu \\ Key Laboratory of Molecular Medicine, \\ Department of Biochemistry and Molecular Biology, Ministry of Education, \\ Shanghai Medical College, Fudan University, Shanghai, P.R. China \\ Corresponding author: M. Yu \\ E-mail: minyu@shmu.edu.cn
}

Genet. Mol. Res. 10 (4): 4147-4157 (2011)

Received February 15, 2011

Accepted June 15, 2011

Published October 31, 2011

DOI http://dx.doi.org/10.4238/2011.October.31.2

\begin{abstract}
Tissue factor (coagulation factor III) is a cell surface receptor for coagulation factor VII/VIIa; it was initially recognized as an initiator of the extrinsic coagulation pathway. Recently, the zebrafish tissue factor gene $(T F)$ has been cloned. Paralogs encode coagulation factors IIIa and IIIb; both show remarkable sequence identity to the human and mouse coagulation factor III gene. It has been reported that $T F$ could have additional properties that are essential for normal embryonic development, since knockout of the murine coagulation factor III gene resulted in $90 \%$ embryonic lethality. We examined the role of coagulation factor IIIb $(f 3 b)$ during zebrafish embryonic development. Expression analysis revealed that endogenous $f 3 b$ was chronologically expressed in the pectoral fins and in the vicinity of the pharynx. Knockout of $f 3 b$ by injection of an $f 3 b$ morpholino at the oneto-two cell stage caused distinctive morphological defects in embryos, including edema in the fourth brain ventricle at early embryonic stages and occasional bleeding at later stages. Furthermore, $f 3 b$ morphants displayed abnormal vascular patterning. We conclude that $f 3 b$ is required for brain vascular development and for development of part of the somatic vasculature during embryogenesis in the zebrafish.
\end{abstract}

Key words: Tissue factor; Vasculature; Brain development; Zebrafish 


\section{INTRODUCTION}

Tissue factor (TF) is a transmembrane glycoprotein that shares structural homology with class II cytokine receptors (Bazan, 1990). The human $T F$ gene has been cloned (Spicer et al., 1987; Morrissey et al., 1987; Mackman et al., 1989) and is localized on chromosome 1 (p21p22). The 6 exons are translated into a 263 -amino acid protein with a 219 -amino acid extracellular domain, a 23-amino acid transmembrane region, and a 21-amino acid intracellular domain. Also known as coagulation factor III, TF is the cell surface receptor for factor VII (FVII), which is responsible for triggering blood coagulation (Nemerson, 1988; Carson and Brozna, 1993). Tissue factor is involved in thrombosis and inflammation associated with sepsis, atherosclerosis, tumor progression, and embryogenesis (Pawlinski and Mackman, 2004; Chen and Dorling, 2009).

Constitutive expression of the TF protein and mRNA was confined to stromal fibroblasts in vascular adventitia and organ capsules, to epithelial cells in the skin and mucosa, to stromal cells in the endometrium, and to astrocytes in the brain (Edgington et al., 1991; Osterud et al., 1995). In situ hybridization studies in normal murine tissues revealed a similar pattern, but TF mRNA was also expressed in the embryonic epithelia from E9.5-12.5, including cornea and pharyngeal epithelium (Mackman et al., 1993; Soifer et al., 1994). It has been reported that humans cannot survive without TF (Tuddenham et al., 1995), and homozygous knockout of tissue factor in mouse embryos resulted in approximately $90 \%$ embryonic lethality by E10.5 (Carmeliet et al., 1996; Toomey et al., 1996). Therefore, TF is essential for embryonic development, but its specific functions are unknown.

The study of zebrafish embryogenesis has yielded many insights into the development of the embryonic vasculature. Zebrafish embryos develop externally, are nearly transparent, and are thin enough for diffusion-mediated oxygenation from the medium. In fact, zebrafish can survive the first week of development without a functional vasculature or heart beat, allowing a detailed analysis even in animals with severe cardiovascular defects. By contrast, avian and mammalian embryos die rapidly in the absence of a functional cardiovascular system (Stainier et al., 1995, 2001; Sehnert et al., 2002).

The zebrafish coagulation factor IIIb $(f 3 b)$ gene has been cloned (Stein et al., 2007) and shows significant homology with the human and mouse $T F$ gene. We demonstrate that endogenous $f 3 b$ is expressed in the pectoral fins and in the vicinity of the pharynx. Knockdown of $f 3 b$ caused distinct morphological defects in embryos; early stage embryos exhibited edema in the fourth brain ventricle, while later stage embryos showed signs of regional hemorrhage. Furthermore, $f 3 b$ morphants displayed abnormal vascular patterning as revealed by fluorescence angiography.

\section{MATERIAL AND METHODS}

\section{Zebrafish strains and maintenance}

Zebrafish (AB strain) embryos were collected and staged as previously described (Kimmel et al., 1995; Westerfield, 2000). In order to block pigment formation in embryos, $0.003 \%$ phenylthiourea was added to the medim at $18 \mathrm{hpf}$ (hours post-fertilization) and replenished every $24 \mathrm{~h}$ thereafter. Embryos were fixed in 4\% paraformaldehyde-phosphate-buffered saline at $4{ }^{\circ} \mathrm{C}$ and stored in $100 \%$ methanol at $-20^{\circ} \mathrm{C}$ prior to in situ hybridization. 


\section{Reverse transcription-polymerase chain reaction (RT-PCR)}

Total RNA was extracted from 25 to 30 embryos using TRIzol Reagent (Invitrogen, USA) according to manufacturer instructions. One microgram of total RNA was used as the template in a $20-\mu \mathrm{L}$ RT-PCR mixture using a one-step RT-PCR kit (Qiagen, Germany). The RT-PCR conditions were as described by Berghmans et al. (2005) except for a change in annealing temperature, which depended on the Tm value of the primers.

\section{Whole-mount in situ hybridization}

Whole-mount in situ hybridization experiments using the combination of digoxigenin-labeled antisense RNA probe and a digoxigenin/alkaline phosphatase-conjugated antibody (Roche) were performed as previously described (Westerfield, 2000). For the $f 3 b$ and $f k l$ (Yamaguchi et al., 1993) antisense probes, the NBT/BCIP coloring reactions were carried out at about $20^{\circ} \mathrm{C}$ for $2 \mathrm{~h}$.

\section{Morpholino oligonucleotide injection}

Morpholinos (MOs) were obtained from Gene Tools, LLC (USA). MOs targeting the sequences flanking the initiation codon $\left(\mathrm{TF}_{\mathrm{ATG}}-\mathrm{MO}\right)$ or targeting the splice junctions $\left(\mathrm{TF}_{\mathrm{SPL}}-\mathrm{MO}\right)$ of the zebrafish $f 3 b$ gene were injected into embryos at 1- to 4-cell stage. In our experiments, we chose the second spliceosome that consists of the end of exon 2 and the beginning of intron 2 as the $\mathrm{MO}$ targeting site. Either $\mathrm{TF}_{\mathrm{ATG}}-\mathrm{MO}$ or $\mathrm{TF}_{\mathrm{SPL}}-\mathrm{MO}$ at 0.5 $\mathrm{mg} / \mathrm{mL}$ (4 ng/embryo) was injected into the blastomere of one- or two-cell stage embryos. Control MO was injected at the same volume. The sequences of the morpholinos are listed in Table 1.

\begin{tabular}{ll} 
Table 1. Sequences of oligos used. & \\
\hline Oligo & Sequence \\
\hline Morpholinos $^{\text {TFb }}{ }_{\text {ATG }}$ & \\
TFb & 5'-TACAGTCTGAATTCCCATCGTTGGT-3' \\
Random sequence & 5'-GATATTTATGTACTTGCCTTGAGAA-3' \\
Primers for the cloning of TFb mRNA in riboprobe synthesis & 5'-CCTCTTACCTCAGTTACAATTTATA-3' \\
TFb-CDS-1 & \\
TFb-CDS-r & 5'-GGGCAAACTTACCAAAGCAA-3' \\
Primers for the cloning of TFb-EGFP construct synthesis & 5'-TTTCCTGTGCTTCCAGCTTT-3' \\
TFb-egfp-1 & \\
TFb-egfp-r & 5'-CCGCTCGAGGCAGGACGGATTTATTGA-3' \\
Primers for RT-PCR for TFb mRNA in splice-site morpholino study & 5'-CGCGGATCCTCCTCATCCAGTCGGTA-3' \\
TFb-SS-1 & \\
TFb-SS-r & 5'-GTAATATGTTCGGCACT-3' \\
\hline
\end{tabular}

\section{Construction of $f 3 b$-EGFP plasmids}

The 87-bp untranslated region and the full-length cDNA of the zebrafish $f 3 b$ were generated by PCR with specially designed primers (Table 1). This PCR product was digested with BamHI and XhoI (New England Biolaboratory, Inc.), gel-purified (QIAQuickgel extrac- 
tion kit), and subcloned into the pEGFP-N1 plasmid (CLONTECH Laboratories, Inc.). The recombinant plasmid was identified by restriction digestion analysis, and sequence accuracy was confirmed by automated DNA sequencing. The $\mathrm{TF}_{\mathrm{ATG}_{\mathrm{G}}}-\mathrm{MO}$ (4 ng $\mathrm{MO} / \mathrm{embryo}$ ) or plasmid DNA (50-100 pg/embryo) was microinjected into embryos at the one- or two-cell stage.

\section{Fluorescence angiography}

Zebrafish at 48 or $72 \mathrm{hpf}$ were anesthetized with a standard solution of $0.02 \%$ tricaine (w/v; tricaine methane sulfonate, MS-222; Sigma, A5040) for 2-4 min. Fish were then transferred to a slotted $1 \%$ agarose gel with its ventral side up. About $5 \mu$ L FITC-Dextran (50 $\mathrm{mg} / \mathrm{mL}$; Sigma-Aldrich Co.) was delivered into the heart using standard microinjection equipment. A mounted fish was placed under a fluorescent microscope (Olympus BX61) to assess vascular patterning.

\section{Photography}

Stained embryos were examined with Olympus BX61 and SZX12 microscopes and photographed with a DP70 digital camera. Images were processed using the Adobe Photoshop software.

\section{RESULTS}

\section{Expression of the $f 3 b$ gene in zebrafish embryos}

RT-PCR was performed on zebrafish embryos at a series of stages: 6-somite $(12 \mathrm{~h}$ hpf), 18-somite (18 hpf), prim-5 (24 hpf), prim-25 (36 hpf), long pec (48 hpf), and protrudingmouse (72 hpf). The $f 3 b$ mRNA was detected as early as $18 \mathrm{hpf}$ (Figure 1). Whole-mount in $s i t u$ hybridization with the $f 3 b$ antisense probe was performed to examine regional expression of the $f 3 b$ mRNA in zebrafish embryos. Staining was detected in the pectoral fin as of $36 \mathrm{hpf}$ and in the vicinity of the pharynx by $72 \mathrm{hpf}$ (Figure 2).

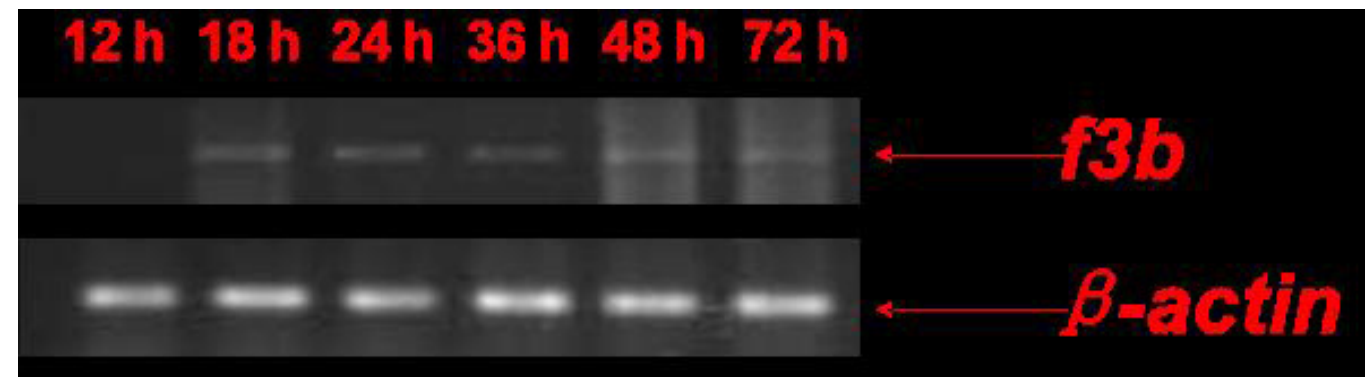

Figure 1. RT-PCR analysis showing $f 3 b$ expression. RT-PCR analysis was performed with RNA samples isolated from different embryonic stages. $f 3 b$-specific products were amplified from RNA isolated from 12-72 hpf (hours post-fertilization) whole embryos. The $\beta$-actin primers amplified a single fragment in all samples and $\beta$-actin acted as the internal control. 


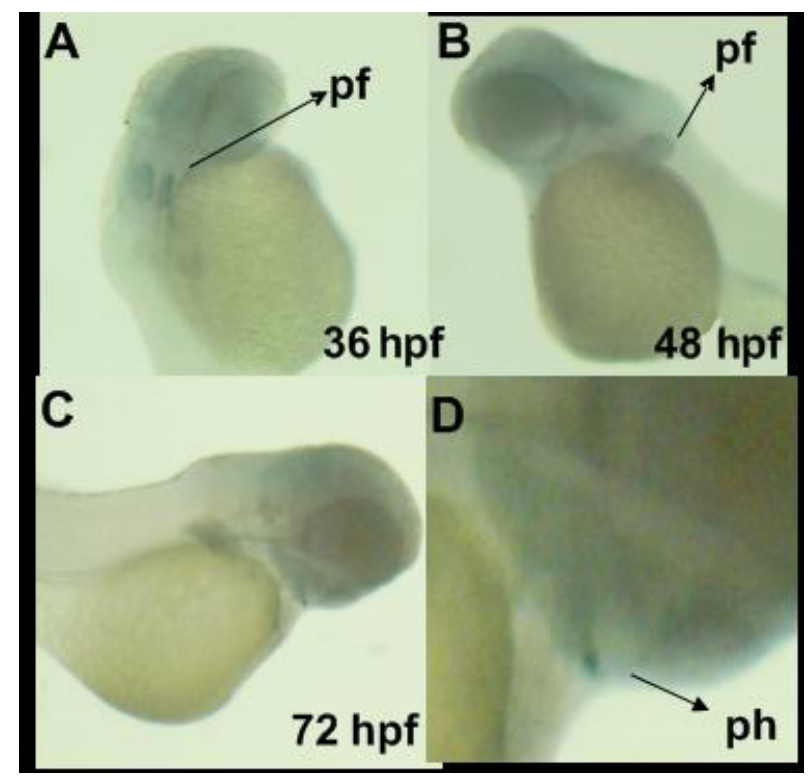

Figure 2. Expression pattern of $f 3 b$ during zebrafish embryogenesis. A.-D. Lateral view. Embryonic stages are indicated at bottom right. $f 3 b$ was also expressed in pf (arrow) from $36 \mathrm{hpf}$ (hours post-fertilization) on (A, B), and $\mathrm{ph}$ (arrow) at $72 \mathrm{hpf}(\mathrm{C})$. (D) High magnification of C. pf = pectoral fin; ph = pharynx.

\section{Characterization of the $f 3 b$ morphant phenotype}

The role of $f 3 b$ during embryonic development was investigated by mRNA knockdown using MOs. Most embryos had a relatively normal morphology at early stage embryo-

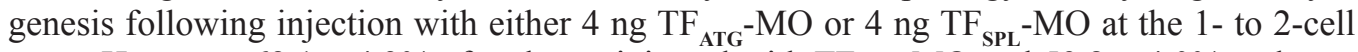
stage. However, $62.1 \pm 4.3 \%$ of embryos injected with $\mathrm{TF}_{\mathrm{ATG}}-\mathrm{MO}$ and $59.8 \pm 4.0 \%$ embryos injected with $\mathrm{TF}_{\mathrm{SPL}}-\mathrm{MO}$ manifested edema in the fourth brain ventricle by $24 \mathrm{hpf}$ (data not shown). By 36 hpf, some embryos demonstrated occasional bleeding at different sites (but mainly in the brain). Embryos injected with $\mathrm{TF}_{\mathrm{ATG}}-\mathrm{MO}$ showed bleeding in pharynx and brain at $72 \mathrm{hpf}$ while embryos injected with scrambled nucleotides at the 1- to 2-cell stage showed no abnormalities during embryogenesis. Similar aberrant phenotypes were also observed in embryos injected with either $\mathrm{TF}_{\mathrm{ATG}}-\mathrm{MO}$ or $\mathrm{TF}_{\mathrm{SPL}}-\mathrm{MO}$ at various doses by $72 \mathrm{hpf}$ but not in wild-type embryos injected with a random MO sequence (Table 2, Figure 3).

To confirm that $\mathrm{TF}_{\mathrm{ATG}_{\mathrm{TG}}}-\mathrm{MO}$ suppressed the $f 3 b$ mRNA, we measured the suppression of a fluorescent reporter linked to the transcript. At the 1- to 2-cell stage, $4 \mathrm{ng} \mathrm{TF}_{\mathrm{ATG}}-\mathrm{MO}$ or a random control sequence was co-injected with a green fluorescent protein (GFP) reporter containing the partial 87-bp untranslated region and the full-length cDNA of $f 3 b$ (Figure 4, I). The $\mathrm{TF}_{\mathrm{ATG}}-\mathrm{MO}$ specifically knocked down the expression of GFP from this RNA transcript in $100 \%$ of embryos at $9 \mathrm{hpf}(\mathrm{N}=47)$ as revealed by the lack of green fluorescent emission (Figure 4, IIC). In comparison, there was no detectable knockdown of GFP when the GFP-f $3 b$ transcript was co-injected with a random control $\mathrm{MO}(\mathrm{N}=63)$ (Figure 4, IIB). Thus, $\mathrm{TF}_{\mathrm{ATG}^{-}}{ }^{-}$ MO specifically inhibited the production of the EGFP-f3b protein.

To test the efficacy of the $\mathrm{TF}_{\mathrm{SPL}}-\mathrm{MO}$, we conducted RT-PCR. Embryos injected with 
$4 \mathrm{ng} \mathrm{TF}_{\mathrm{SPL}}-\mathrm{MO}$ were collected and RT-PCR was performed to confirm the defective splicing conferred by the missing exon 2. Indeed, bidirectional DNA sequencing revealed lower PCR transcript levels in injected embryos (Figure 4, III).

\begin{tabular}{|c|c|c|c|c|}
\hline MO (4 ng) & Times & Normal & Abnormal & Death \\
\hline \multirow{4}{*}{$\mathrm{TF}_{\mathrm{ATG}}-\mathrm{MO}$} & 1 & $19 / 120$ & $78 / 120$ & $23 / 120$ \\
\hline & 2 & $23 / 120$ & $72 / 120$ & $25 / 120$ \\
\hline & 3 & $17 / 120$ & $75 / 120$ & $28 / 120$ \\
\hline & $\operatorname{mean} \pm \mathrm{SD}$ & $16.39 \pm 2.55 \%$ & $62.50 \pm 2.50 \%$ & $21.11 \pm 2.10 \%$ \\
\hline \multirow{4}{*}{$\mathrm{TF}_{\mathrm{SPL}}-\mathrm{MO}$} & 1 & $21 / 120$ & $71 / 120$ & $28 / 120$ \\
\hline & 2 & $25 / 120$ & $68 / 120$ & $27 / 120$ \\
\hline & 3 & $19 / 120$ & $72 / 120$ & $29 / 120$ \\
\hline & mean $\pm \mathrm{SD}$ & $18.06 \pm 2.55 \%$ & $58.61 \pm 1.73 \%$ & $23.33 \pm 0.83 \%$ \\
\hline \multirow[t]{4}{*}{ Control-MO } & 1 & $107 / 120$ & $1 / 120$ & $12 / 120$ \\
\hline & 2 & $104 / 120$ & $2 / 120$ & $14 / 120$ \\
\hline & 3 & $103 / 120$ & $1 / 120$ & $16 / 120$ \\
\hline & mean $\pm \mathrm{SD}$ & $87.22 \pm 1.73 \%$ & $1.11 \pm 0.48 \%$ & $11.67 \pm 1.67 \%$ \\
\hline
\end{tabular}

The total number of animals (120) was scored at $48 \mathrm{hpf}$, and phenotypes were separated into three categories: normal, abnormal (showing either "brain edema" or "bleeding"), and death. We recorded our results of three randomized controlled trials as shown above.

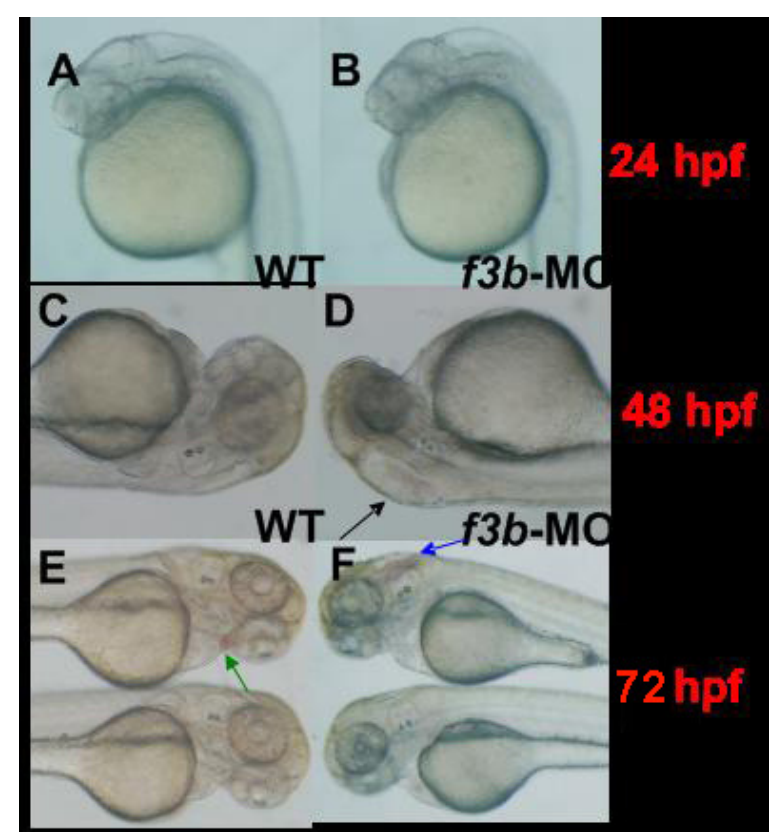

Figure 3. Effects of $f 3 b$ knockdown on embryonic development. A.-F. Lateral view. A.C. Wild type (WT) embryos were injected with random sequence morpholino (MO) at the 1- to 2-cell stage and visualized at 24 hpf (hours post-fertilization; A) and $48 \mathrm{hpf}$ (C). B.D. Embryos injected with $4 \mathrm{ng} \mathrm{TF}_{\mathrm{ATG}}-\mathrm{MO}$ at the 1- to 2-cell stage and photographed at $24 \mathrm{hpf}(\mathrm{B})$ and $48 \mathrm{hpf}(\mathrm{D})$. Note that most of the embryos injected with $\mathrm{TF}_{\mathrm{ATG}}-\mathrm{MO}$ showed edema in the fourth brain ventricle at $24 \mathrm{hpf}$ (B), and occasional bleeding (black arrow) at $48 \mathrm{hpf}$ (D). E.F. Embryos injected with $\mathrm{TF}_{\mathrm{ATG}}-\mathrm{MO}$ (upper embryo in both $\mathrm{E}$ and $\mathrm{F}$ ) showed bleeding around the pharynx (green arrow) and in the brain (blue arrow) at $72 \mathrm{hpf}$. In contrast, WT embryos showed no vascular abnormalities (lower embryo in both E and F). 

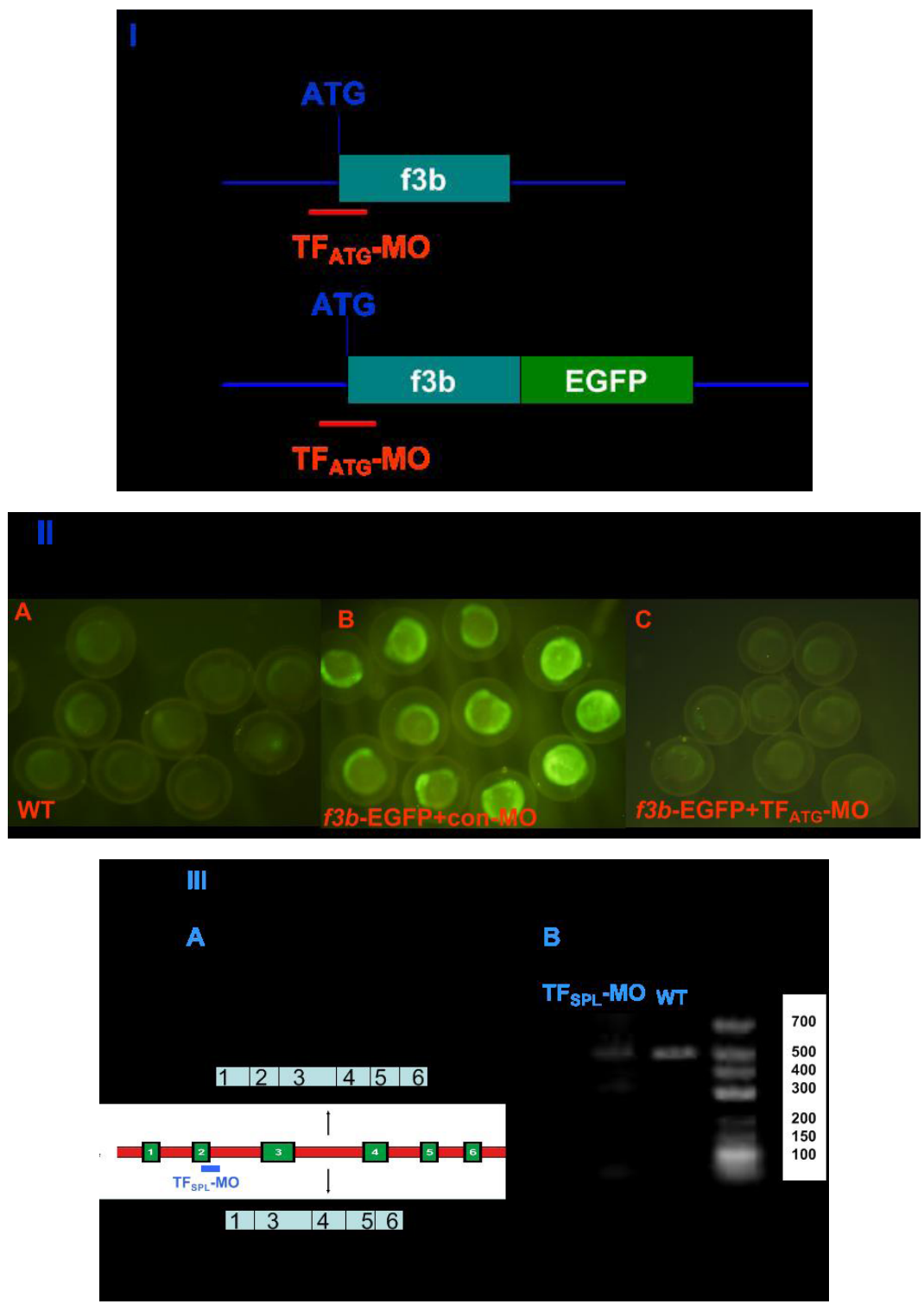

Figure 4. Specificity of $f 3 b$ knockdown. I. Scheme showing the position of the $\mathrm{TF}_{\mathrm{ATG}_{\mathrm{T}}}-\mathrm{MO}$ of the zebrafish $f 3 b$ mRNA. To test the ability of $\mathrm{TF}_{\mathrm{ATG}}-\mathrm{MO}$ to knock down expression of its target sequence, an EGFP reporter construct was produced. IIA-C. Fluorescence microscopy images in $9 \mathrm{hpf}$ zebrafish embryos. IIB. Fluorescence microscopy image of zebrafish embryos co-injected with the $f 3 b$-GFP plasmid and a random control MO. Intense GFP emission (green) was detected throughout the injected embryos. IIC. Fluorescence microscopy image of zebrafish embryos co-injected with the $f 3 b$-GFP plasmid and $\mathrm{TF}_{\mathrm{ATG}}-\mathrm{MO}$. Note the absence of detectable GFP emission in all embryos. IIIA. Scheme showing the position of the $\mathrm{TF}_{\text {SPL }^{-}}-\mathrm{MO}$ of the zebrafish $f 3 b$ mRNA. IIIB. Molecular targeting was confirmed using RT-PCR and showed that injected embryos contained less $f 3 b$ mRNA than uninjected embryos. TF $=$ tissue factor; $f 3 b=$ coagulation factor IIIb; $\mathrm{MO}=$ morpholino; $\mathrm{WT}=$ wild-type embryo; EGFP = enhanced green fluorescent protein; $\mathrm{TF}_{\mathrm{ATG}}-\mathrm{MO}$ and $\mathrm{TF}_{\mathrm{SPL}}-\mathrm{MO}=\mathrm{MOs}$ targeting the sequences flanking the initiation codon and the splice junctions, respectively. 


\section{Effects of $f 3 b$ knockdown on vasculature}

The $f 3 b$ morphants showed extravasation of blood cells from different vessels. Therefore, we first examined the effects of $f 3 b$ knockdown on vascular formation by hybridization with $f k 1$, a transcript from a gene that encodes an endothelial-specific receptor of vascular endothelial growth factor (VEGF). During embryogenesis, $f k 1$ expression is first detected in hemangioblasts, the common precursor of the endothelial and blood lineages, and remains active in endothelial cells during vascular formation (Yamaguchi et al., 1993; Millauer et al., 1993). By whole-mount in situ hybridization with $f k 1$, we demonstrated a significant reduction in the intermediate cell mass and in brains of $f 3 b$ morphants. In control embryos, flk 1 expression revealed a ringed-vascular structure in the brain at $28 \mathrm{hpf}$. However, this structure was deformed in $f 3 b$ morphants (Figure 5). We also examined the vasculature of zebrafish in late embryogenesis by fluorescence angiography, and found that the $f 3 b$ morphants displayed a "string-of-beads" vascular structure at 48 hpf compared with control embryos. By 72 hpf, $f 3 b$ morphants showed extravasation of blood cells around the abnormal vasculature (Figure 5).

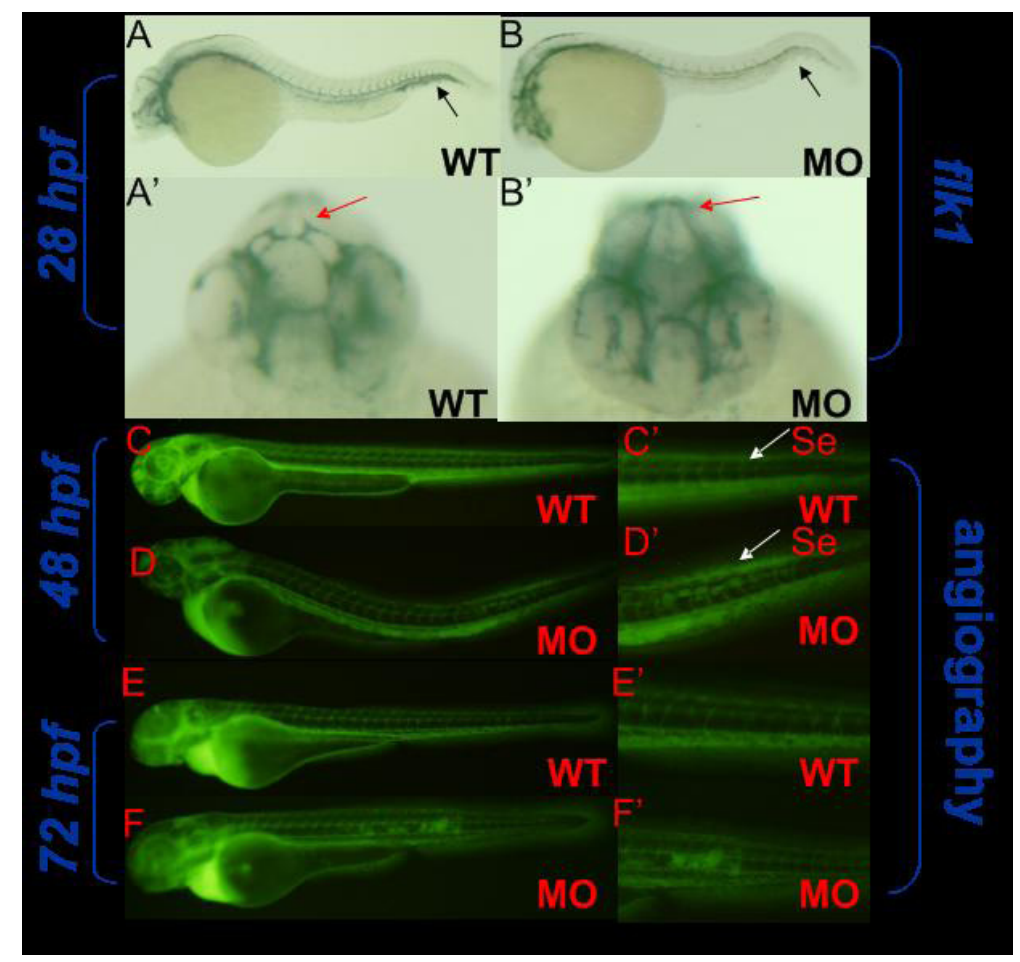

Figure 5. $f 3 b$ is required for proper vascular patterning. A.B. Lateral view. A'.B'. Overhead view. A.A'. Wholemount in situ hybridization with $f k 1$ at $28 \mathrm{hpf}$ (hours post-fertilization) in a control embryo. B.B'. Whole-mount in situ hybridization with $f k l$ at $28 \mathrm{hpf}$ in an $f 3 b$ morphant, showing significant reduction in intermediate cell mass (black arrow) and altered expression in brain (red arrow). C.-F. Fluorescence angiography at 48/72 hpf in the control embryo (C, E) and $f 3 b$ morphants $(\mathrm{D}, \mathrm{F})$. C', D', E', F' are high magnification images from C, D, E, and F. Note that the somatic vessel (white arrow) has a "string of beads" structure at $48 \mathrm{hpf}$ and hemorrhage at $72 \mathrm{hpf}$ in $f 3 b$ morphants but not in control embryos. WT $=$ wild-type embryos; $\mathrm{MO}=$ morpholino. 


\section{DISCUSSION}

In this study, we investigated the temporal and spatial expression pattern of zebrafish $f 3 b$ and the phenotype conferred by $f 3 b$ knockdown. Knockdown of $f 3 b$ using MOs gave rise to embryos with edema in the fourth brain ventricle and occasional bleeding. In contrast, $f 3 b$ hypofunction did not cause gross developmental delay.

\section{What is known about $f 3 a$}

We searched a zebrafish library and found the cDNA sequence of the zebrafish $T F$ gene with two homologues, $f 3 a$ (GenBank ID No. XM_690549) and $f 3 b$ (GenBank ID No. NM_001017728). The sequence of the $\mathrm{f} 3 \mathrm{a}$ protein was predicted by automated computational analysis, but knockdown using MO did not yield a measurable phenotype, suggesting that $f 3 a$ may require more accurate sequencing.

\section{The specificity of gene expression and MO knockdown}

The sequence of the $f 3 b$ mRNA riboprobe was consistent with the cDNA sequence found by Blast search. We then designed two different MOs targeting the $f 3 b$ gene and a control MO. By microinjection into zebrafish at the 1- to 2-cell stage, both of the $f 3 b$ MOs led to the same phenotype, while embryos injected with the control MO appeared normal.

\section{Edema in the fourth brain ventricle}

Knockdown of $f 3 b$ with these MOs yielded embryos with edema in the fourth brain ventricle by the pharyngula period. Moreover, whole-mount in situ hybridization with a probe for the VEGF receptor demonstrated a significant reduction in the intermediate cell mass containing hematopoietic progenitor cells. Knockdown also altered $f 3 b$ expression in the brain in $f 3 b$ morphants, leading to edema. While the mechanism for edema has not been fully explained, the aberrant morphology of the CNS vasculature (the "string of beads" conformation) observed by fluorescence angiography may have produced an obstruction that impeded cerebrospinal circulation, leading to elevated pressure and edema.

\section{The reason for occasional bleeding}

In addition to improper vascular development, knockdown of $f 3 b$ resulted in occasional bleeding at different sites, including the brain and pharynx. Indeed, the high expression of $f 3 b$ at these sites in wild-type embryos underscores the role of $f 3 b$ in angiogenesis within these regions. Under normal physiological conditions, zebrafish show a very low propensity for bleeding because TF initiates the coagulation cascade when the blood vessels are damaged. Therefore, we speculated that knockdown of $f 3 b$ resulted in both abnormal vasculature patterning and reduced coagulation, leading to localized hemorrhage. Microscopic analysis demonstrated aberrant vasculature construction prior to bleeding, indicating that $f 3 b$ participates in both angiogenesis and the coagulation response. This conclusion is consistent with previous observations; mice lacking TF die at E8.5 secondary to insufficient circulation from the yolk 
sac to embryo (Carmeliet et al., 1996). Also, in vitro studies have shown that alternatively spliced TF enhanced the migration and differentiation, but not proliferation, of endothelial cells (He et al., 2008).

In summary, our findings suggest that, in addition to its better known role in coagulation, $f 3 b$ is required for angiogenesis during early zebrafish embryogenesis. However, it still remains unclear how TF affects the differentiation and patterning of endothelial cells or vascular smooth muscle cells. Future studies will be directed toward understanding the underlying molecular mechanisms and could provide valuable insights into angiogenesis and the pathophysiology of sepsis and atherosclerosis.

\section{ACKNOWLEDGMENTS}

Research supported by the National Natural Science Foundation of China (\#30870565). We are grateful to Prof. Houyang Song and Dr. Jingying Hu for constructive advice and critical reading of the manuscript, and to other members of the Song Laboratory for technical assistance.

\section{REFERENCES}

Bazan JF (1990). Structural design and molecular evolution of a cytokine receptor superfamily. Proc. Natl. Acad. Sci. U. S. A. 87: 6934-6938.

Berghmans S, Murphey RD, Wienholds E, Neuberg D, et al. (2005). tp53 mutant zebrafish develop malignant peripheral nerve sheath tumors. Proc. Natl. Acad. Sci. U. S. A. 102: 407-412.

Carmeliet P, Mackman N, Moons L, Luther T, et al. (1996). Role of tissue factor in embryonic blood vessel development. Nature 383: 73-75.

Carson SD and Brozna JP (1993). The role of tissue factor in the production of thrombin. Blood Coagul. Fibrinolysis 4: 281-292.

Chen D and Dorling A (2009). Critical roles for thrombin in acute and chronic inflammation. J. Thromb. Haemost. 7 (Suppl 1): 122-126.

Edgington TS, Mackman N, Brand K and Ruf W (1991). The structural biology of expression and function of tissue factor. Thromb. Haemost. 66: 67-79.

He Y, Chang G, Zhan S, Song X, et al. (2008). Soluble tissue factor has unique angiogenic activities that selectively promote migration and differentiation but not proliferation of endothelial cells. Biochem. Biophys. Res. Commun. 370: 489-494.

Kimmel CB, Ballard WW, Kimmel SR, Ullmann B, et al. (1995). Stages of embryonic development of the zebrafish. Dev. Dyn. 203: 253-310.

Mackman N, Morrissey JH, Fowler B and Edgington TS (1989). Complete sequence of the human tissue factor gene, a highly regulated cellular receptor that initiates the coagulation protease cascade. Biochemistry 28: 1755-1762.

Mackman N, Sawdey MS, Keeton MR and Loskutoff DJ (1993). Murine tissue factor gene expression in vivo. Tissue and cell specificity and regulation by lipopolysaccharide. Am. J. Pathol. 143: 76-84.

Millauer B, Wizigmann-Voos S, Schnurch H, Martinez R, et al. (1993). High affinity VEGF binding and developmental expression suggest Flk-1 as a major regulator of vasculogenesis and angiogenesis. Cell 72: 835-846.

Morrissey JH, Fakhrai H and Edgington TS (1987). Molecular cloning of the cDNA for tissue factor, the cellular receptor for the initiation of the coagulation protease cascade. Cell 50: 129-135.

Nemerson Y (1988). Tissue factor and hemostasis. Blood 71: 1-8.

Osterud B, Bajaj MS and Bajaj SP (1995). Sites of tissue factor pathway inhibitor (TFPI) and tissue factor expression under physiologic and pathologic conditions. On behalf of the Subcommittee on Tissue Factor Pathway Inhibitor (TFPI) of the Scientific and Standardization Committee of the ISTH. Thromb. Haemost. 73: 873-875.

Pawlinski R and Mackman N (2004). Tissue factor, coagulation proteases, and protease-activated receptors in endotoxemia and sepsis. Crit. Care Med. 32: S293-S297.

Sehnert AJ, Huq A, Weinstein BM, Walker C, et al. (2002). Cardiac troponin T is essential in sarcomere assembly and 
cardiac contractility. Nat. Genet. 31: 106-110.

Soifer SJ, Peters KG, O'Keefe J and Coughlin SR (1994). Disparate temporal expression of the prothrombin and thrombin receptor genes during mouse development. Am. J. Pathol. 144: 60-69.

Spicer EK, Horton R, Bloem L, Bach R, et al. (1987). Isolation of cDNA clones coding for human tissue factor: primary structure of the protein and cDNA. Proc. Natl. Acad. Sci. U. S. A. 84: 5148-5152.

Stainier DY (2001). Zebrafish genetics and vertebrate heart formation. Nat. Rev. Genet. 2: 39-48.

Stainier DY, Weinstein BM, Detrich HW III, Zon LI, et al. (1995). Cloche, an early acting zebrafish gene, is required by both the endothelial and hematopoietic lineages. Development 121: 3141-3150.

Stein C, Caccamo M, Laird G and Leptin M (2007). Conservation and divergence of gene families encoding components of innate immune response systems in zebrafish. Genome Biol. 8: R251.

Toomey JR, Kratzer KE, Lasky NM, Stanton JJ, et al. (1996). Targeted disruption of the murine tissue factor gene results in embryonic lethality. Blood 88: 1583-1587.

Tuddenham EG, Pemberton S and Cooper DN (1995). Inherited factor VII deficiency: genetics and molecular pathology. Thromb. Haemost. 74: 313-321.

Westerfield M (2000). The Zebrafish Book: A Guide for the Laboratory Use of Zebrafish (Danio rerio). University of Oregon Press, Oregon.

Yamaguchi TP, Dumont DJ, Conlon RA, Breitman ML, et al. (1993). flk-1, an flt-related receptor tyrosine kinase is an early marker for endothelial cell precursors. Development 118: 489-498. 